\begin{tabular}{|c|c|c|}
\hline \multirow[t]{2}{*}{7} & $\begin{array}{l}\text { International Journal of Current Research in } \\
\text { Biosciences and Plant Biology }\end{array}$ & \\
\hline & Volume $4 \bullet$ Number 8 (August-2017) • ISSN: 2349-8080 (Online) & \\
\hline $\begin{array}{l}\text { EXCELLENT } \\
\text { PUBLISHERS }\end{array}$ & Journal homepage: www.ijcrbp.com & www.jictip. coum \\
\hline
\end{tabular}

\title{
Synthesis and Characterization of Some Transition and Non-Transition Metal Complexes with New Cyclic and Open Series Containing (N, O) as Donor Atoms
}

\author{
Hikmat Ali Mohamad ${ }^{1}$ and Karam Salahaddin Shreef ${ }^{2} *$ \\ ${ }^{I}$ College of Education, University of Salahaddin, Erbil, Iraq \\ ${ }^{2}$ College of Science, University of Mosul, Mosul, Iraq \\ *Corresponding author.
}

\begin{abstract}
Transition and non transition metal ion complexes $\mathrm{Mn}(\mathrm{II}), \mathrm{Fe}(\mathrm{II}), \mathrm{Co}(\mathrm{II}), \mathrm{Ni}(\mathrm{II}), \mathrm{Cu}(\mathrm{II})$, $\mathrm{Zn}(\mathrm{II}), \mathrm{Cd}(\mathrm{II})$ complexes with the $\mathrm{L}=\mathrm{N}^{1}, \mathrm{~N}^{4}$ - bis (4-amino phenyl) succinamaide (L) from the reaction of p-phenylene di amine and diethyl succinate. Which the characterization of the ligand has been done on the basis of micro element analysis, infrared, spectra measurement. The complexes were characterized using IR, UV-Visible, molar conductivity, magnetic susceptibility, C.H.N.Cl analysis, IR spectra data suggest that the ligand was chelated with ionic metals via $\mathrm{O}(\mathrm{C}=\mathrm{O})$ and $\mathrm{N}\left(\mathrm{NH}_{2}\right)$ atoms. On the basis of physicochemical data as well as magnetic moment measurements, octahedral geometries were assigned for the complexes.
\end{abstract}

\section{Article Info}

Accepted: 26 July 2017

Available Online: 06 August 2017

\section{Keywords}

Ligands

Metal complexes

Non transition

Transition

\section{Introduction}

Macro cyclic compound are natural or synthetic polydentate ligands, having the donor atoms. They contain at least three donor atoms and the ring should have at least nine atoms (Lindoy, 1989). The macro cyclic ligand have been attracted, the interesting of the scientists because of their properties, such containing of more than donor atoms and the using of their complexes in different fields (Christensen et al., 1971; Akine et al., 2001). The synthesis of transition and non-transition metal macro cyclic complexes is a continuously developing area of investigation in inorganic and bioorganic chemistry (Beswick et al., 1996). The coordination chemistry of this type of the ligand was attracted the attention of the scientists, because of their role in application in different fields (Delgado, 1995). The earliest known examples of metal complexes of macro cyclic ligand were observed in natural substances, such as the porphyrin ring of the iron containing hem proteins (Lindoy, 1989; Melson, 1979; Goedken, 1979).

The preparation of some macro cyclic complexes containing nitrogen atoms with $\mathrm{Ni}(\mathrm{II})$ has been done as crown ethercomplexes (Lindoy, 1989). In this work the efforts were made to preparation of some complexes from the reaction of $\mathrm{N}^{1}, \mathrm{~N}^{4}$ - bis (4-amino phenyl) succinamide (L)with[Mn(II), Fe(II), Co(II), Ni(II), $\mathrm{Cu}(\mathrm{II}), \mathrm{Zn}(\mathrm{II}), \mathrm{Cd}(\mathrm{II})]$ ions. 


\section{Materials and methods}

All chemicals used were either analytical grade or chemically pure. Conductivity measurement were carried out on $10^{-3} \mathrm{M}$ solution of all complexes in DMF using (Conductivity Meter Model 4510 -JENWAY), IR Spectra were recorded on a Fourier-transform (FT.IR) spectra photo-meter, Tensor $27 \mathrm{Co}$. Bruker in the range $4000-400 \mathrm{~cm}^{-1}$ using $\mathrm{KBr}$ discs.

The C.H.N analysis was carried out by Elemental Combustion System Costech Instrument (EC 4010) in Dicle University in Turkey, the electronic spectra for all complexes were recorded on Shimadzu UV-1650 PC UV-Visible Spectrophotometer (190-1100nm) using $10^{-3}$ $\mathrm{M}$ solution in DMF The magnetic measurement was measured at $25^{\circ} \mathrm{C}$ on the solids by faraday's method using broker B.M6 instrument, University of Mosul, Melting point was measured using Melting PointElectro thermal 9300 Engineering LTD.

\section{General procedure for the synthesis of the ligands}

\section{Synthesis of $\mathbf{N}^{1}, \mathbf{N}^{4}$ - bis (4-amino phenyl) succinamide $(\mathrm{L})$ :}

A solid of P-phenylene diamine (2.16 g, 0.02 mole) was added to a solution of diethyl succinate $(1.74 \mathrm{~g}$, $0.01 \mathrm{~mole}, 5.83 \mathrm{ml})$ in $(10 \mathrm{ml})$ of ethanol, the mixture was refluxed for $7 \mathrm{hrs}$. The light brown precipitate was produced, filtered off, dried, washed with cold ethanol and re-crystallized from ethanol (Scheme 1).

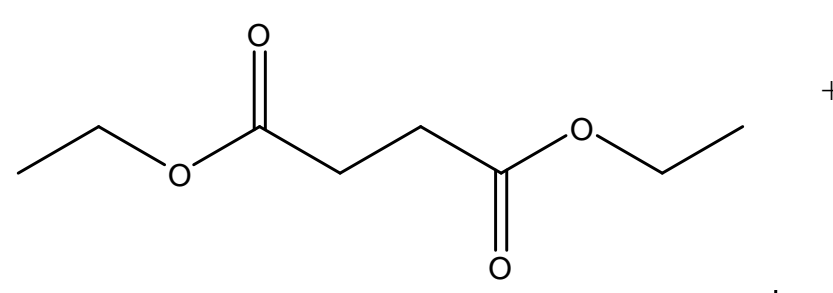

di-ethyl succinate

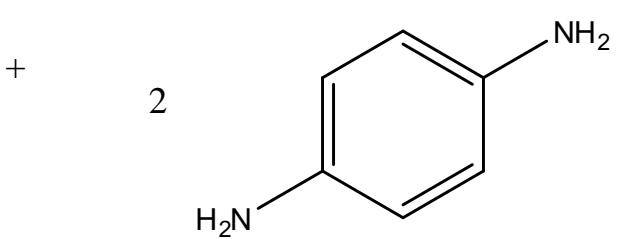

p-phenylene diamine
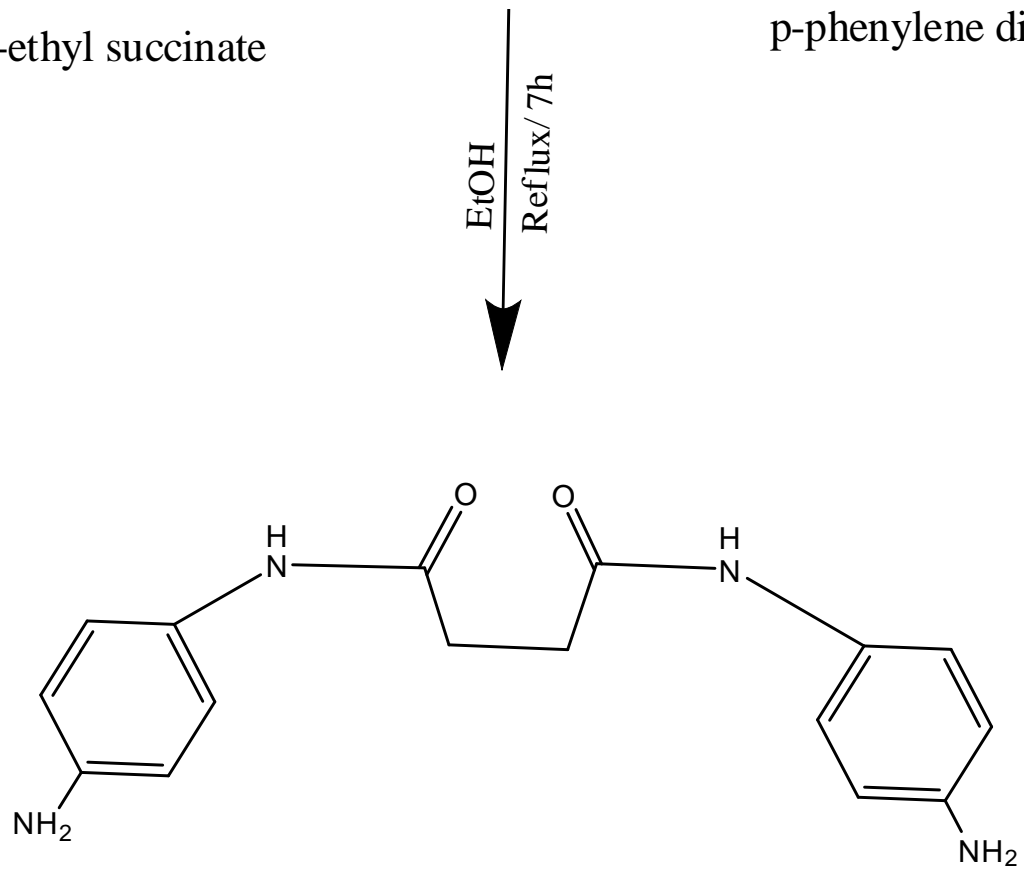

Scheme 1: Preparation of $\mathrm{N}^{1}, \mathrm{~N}^{4}$-bis (4-aminophenyl) succinamide (L).

General procedure for the synthesis of metals complexes: $\left[\mathrm{M}_{2}\left(\mathrm{~L}_{1}\right)\left(\mathrm{H}_{2} \mathrm{O}\right)_{4} \mathrm{Cl4}\right]$

A solution salts $\left(\mathrm{Zg}, 0.001\right.$ mole) of $\mathrm{MCl}_{2} . \mathrm{X} \mathrm{H}_{2} \mathrm{Oin}$ hot ethanol $(10 \mathrm{ml})$ was added to a solution of $(0.298 \mathrm{~g}$,
0.001 mole ) of $\mathrm{L}$ in $(10 \mathrm{ml})$ of ethanol.

The mixture was refluxed for $3 \mathrm{hrs}$ to give colored precipitates, were filtered off, washed with ethanol, dried in oven at $80^{\circ} \mathrm{C}$ for $5 \mathrm{hrs}$ (Scheme 2 and Table 1). 


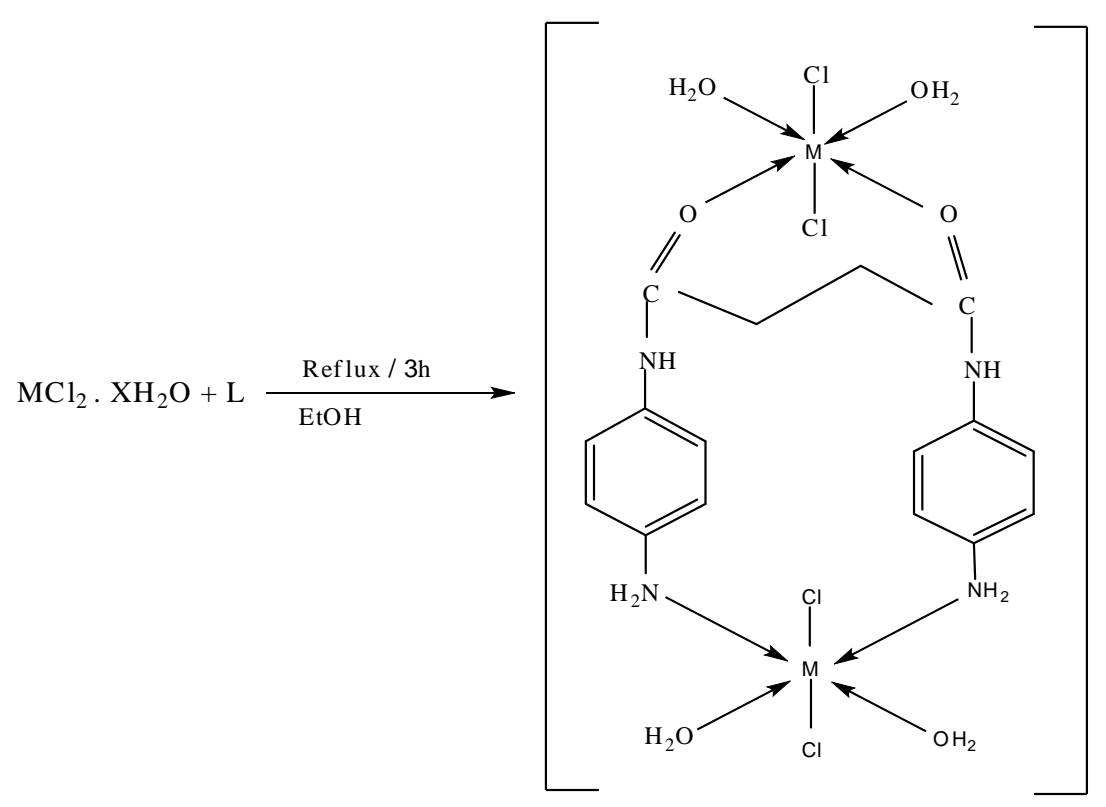

Scheme 2: Preparation of $\left[\mathrm{M}_{2}\left(\mathrm{~L}_{1}\right)\left(\mathrm{H}_{2} \mathrm{O}\right)_{4} \mathrm{Cl} 4\right]$ complexes. $\mathrm{M}=\mathrm{Mn}(\mathrm{II}), \mathrm{Fe}(\mathrm{II})$, $\mathrm{Co}(\mathrm{II}), \mathrm{Ni}(\mathrm{II}), \mathrm{Cu}(\mathrm{II}), \mathrm{Zn}(\mathrm{II}), \mathrm{Cd}(\mathrm{II}) . \mathrm{X}=(2-7)$

\section{Synthesis of $\left[\mathrm{M}_{2}(\mathrm{~L})(\gamma-\mathrm{Pic})_{2}\left(\mathrm{H}_{2} \mathrm{O}\right)_{4} \mathrm{Cl}_{2}\right] \mathrm{Cl}_{2}$ :}

To solution of $\left[\mathrm{M}_{2}(\mathrm{~L})\left(\mathrm{H}_{2} \mathrm{O}\right)_{4} \mathrm{Cl} 4\right]$ ( $\mathrm{Zg}, 0.001$ mole ) in $(10 \mathrm{ml})$ hot ethanol $(0.186 \mathrm{~g}, 0.002 \mathrm{~mol})$ of $\gamma$-Picolinein
$(5 \mathrm{ml})$ of hot ethanol was added.

The mixture was stirred for 30 minutes to give colored complexes (Scheme 3 and Table 2).

Scheme 3: Preparation of $\left[\mathrm{M}_{2}(\mathrm{~L})(\gamma \text {-Pic })_{2}\left(\mathrm{H}_{2} \mathrm{O}\right)_{4} \mathrm{Cl}_{2}\right] \mathrm{Cl}_{2}$ complexes $\mathrm{M}=\mathrm{Mn}(\mathrm{II}), \mathrm{Fe}(\mathrm{II})$, $\mathrm{Co}(\mathrm{II}), \mathrm{Ni}(\mathrm{II}), \mathrm{Cu}(\mathrm{II}), \mathrm{Zn}(\mathrm{II}), \mathrm{Cd}(\mathrm{II})$. 
Table 1. Weight of metal salts of the prepared complexes.

\begin{tabular}{ll}
\hline Chemical Formula of salts & Weight $\mathbf{Z}(\mathbf{g})$ \\
\hline $\mathrm{MnCl}_{2} \cdot 4 \mathrm{H}_{2} \mathrm{O}$ & 0.198 \\
$\mathrm{FeCl}_{2} \cdot 7 \mathrm{H}_{2} \mathrm{O}$ & 0.234 \\
$\mathrm{CoCl}_{2} \cdot 6 \mathrm{H}_{2} \mathrm{O}$ & 0.238 \\
$\mathrm{NiCl}_{2} \cdot 6 \mathrm{H}_{2} \mathrm{O}$ & 0.238 \\
$\mathrm{CuCl}_{2} \cdot 2 \mathrm{H}_{2} \mathrm{O}$ & 0.170 \\
$\mathrm{ZnCl}_{2} \mathrm{XH}_{2} \mathrm{O}$ & 0.244 \\
$\mathrm{CdCl}_{2} \cdot 21 / 2 \mathrm{H}_{2} \mathrm{O}$ & 0.228 \\
\hline
\end{tabular}

Table 2. Weight of $\mathrm{M}_{2}(\mathrm{~L})\left(\mathrm{H}_{2} \mathrm{O}\right)_{4} \mathrm{Cl}_{4}$.

\begin{tabular}{ll}
\hline Chemical formula & Weight $\mathbf{Z}(\mathbf{g})$ \\
\hline$\left[\mathrm{Mn}_{2}(\mathrm{~L})\left(\mathrm{H}_{2} \mathrm{O}\right)_{4} \mathrm{Cl}_{4}\right]$ & 0.622 \\
{$\left[\mathrm{Fe}_{2}(\mathrm{~L})\left(\mathrm{H}_{2} \mathrm{O}\right)_{4} \mathrm{Cl}_{4}\right]$} & 0.623 \\
{$\left[\mathrm{Co}_{2}(\mathrm{~L})\left(\mathrm{H}_{2} \mathrm{O}\right)_{4} \mathrm{Cl}_{4}\right]$} & 0.630 \\
{$\left[\mathrm{Ni}_{2}(\mathrm{~L})\left(\mathrm{H}_{2} \mathrm{O}\right)_{4} \mathrm{Cl}_{4}\right]$} & 0.629 \\
{$\left[\mathrm{Cu}_{2}(\mathrm{~L})\left(\mathrm{H}_{2} \mathrm{O}\right)_{4} \mathrm{Cl}_{4}\right]$} & 0.639 \\
{$\left[\mathrm{Zn}_{2}(\mathrm{~L})\left(\mathrm{H}_{2} \mathrm{O}\right)_{4} \mathrm{Cl}_{4}\right]$} & 0.643 \\
{$\left[\mathrm{Cd}_{2}(\mathrm{~L})\left(\mathrm{H}_{2} \mathrm{O}\right)_{4} \mathrm{Cl}_{4}\right]$} & 0.737 \\
\hline
\end{tabular}

Table 3. The physical properties of the prepared compounds.

\begin{tabular}{|c|c|c|c|c|c|c|c|c|c|}
\hline \multirow{2}{*}{$\begin{array}{l}\text { Ligand } \\
\text { No. }\end{array}$} & \multirow{2}{*}{$\begin{array}{l}\text { Chemical } \\
\text { Formula }\end{array}$} & \multirow{2}{*}{$\begin{array}{l}\text { Molar } \\
\text { conduct. } \\
\text { cm }^{2} . \text { ohm }^{-1} \text {. } \\
\text { moll }^{-1}\end{array}$} & \multirow{2}{*}{$\begin{array}{l}\text { M.P } \\
\left({ }^{\circ} \mathbf{C}\right)\end{array}$} & \multirow{2}{*}{ Color } & \multicolumn{5}{|c|}{ Selected IR( $\left(\mathrm{cm}^{-1}\right)$} \\
\hline & & & & & $v(\mathbf{N H})$ & $\delta(\mathbf{N}-\mathbf{H})$ & $v\left(\mathbf{N H}_{2}\right)$ & $v(\mathbf{C}=\mathbf{O})$ & $(\gamma$-Pic $)$ \\
\hline $\mathrm{L}$ & $\mathrm{C}_{16} \mathrm{H}_{18} \mathrm{~N}_{4} \mathrm{O}_{2}$ & ---- & $137-139$ & $\begin{array}{l}\text { Light } \\
\text { Brown }\end{array}$ & $3197(\mathrm{~s})$ & 1311(s) & $3373(\mathrm{~s})$ & $1635(\mathrm{~s})$ & ----- \\
\hline 1 & {$\left[\mathrm{Mn}_{2}(\mathrm{~L})\left(\mathrm{H}_{2} \mathrm{O}\right)_{4} \mathrm{Cl}_{4}\right]$} & 19 & $275^{*}$ & $\begin{array}{l}\text { Dark } \\
\text { purple }\end{array}$ & $3116(w)$ & $1234(w)$ & $3307(\mathrm{~m})$ & $1630(\mathrm{~s})$ & ----- \\
\hline 2 & {$\left[\mathrm{Fe}_{2}(\mathrm{~L})\left(\mathrm{H}_{2} \mathrm{O}\right)_{4} \mathrm{Cl}_{4}\right]$} & 20 & $170^{-} 172$ & $\begin{array}{l}\text { Brown } \\
\text { Yellow }\end{array}$ & $3128(w)$ & $1290(\mathrm{~s})$ & $3327(\mathrm{~s})$ & $1628(\mathrm{~m})$ & ----- \\
\hline 3 & {$\left[\begin{array}{lll}\mathrm{Co}_{2}(\mathrm{~L})\left(\mathrm{H}_{2} \mathrm{O}\right)_{4} & \mathrm{Cl}_{4}\end{array}\right]$} & 12 & $230^{*}$ & $\begin{array}{l}\text { Dark } \\
\text { Green }\end{array}$ & 3033(s) & $1248(w)$ & 3304(m) & 1616(s) & ----- \\
\hline 4 & {$\left[\mathrm{Ni}_{2}(\mathrm{~L})\left(\mathrm{H}_{2} \mathrm{O}\right)_{4} \mathrm{Cl}_{4}\right]$} & 15 & $228-230$ & Green & 3194(m) & 1228(s) & $3314(w)$ & 1618(s) & ---- \\
\hline 5 & {$\left[\mathrm{Cu}_{2}(\mathrm{~L})\left(\mathrm{H}_{2} \mathrm{O}\right)_{4} \mathrm{Cl}_{4}\right]$} & 30 & $\begin{array}{l}300 \\
>\end{array}$ & $\begin{array}{l}\text { Brown } \\
\text { Yellow }\end{array}$ & $3177(w)$ & $1167(w)$ & $3362(w)$ & $1633(s)$ & ----- \\
\hline 6 & {$\left[\mathrm{Zn}_{2}(\mathrm{~L})\left(\mathrm{H}_{2} \mathrm{O}\right)_{4} \mathrm{Cl}_{4}\right]$} & 5 & $200^{*}$ & Pink & 3182(s) & $1244(\mathrm{~s})$ & $3273(\mathrm{~s})$ & $1628(\mathrm{~m})$ & ----- \\
\hline 7 & {$\left[\mathrm{Cd}_{2}(\mathrm{~L})\left(\mathrm{H}_{2} \mathrm{O}\right)_{4} \mathrm{Cl}_{4}\right]$} & 30 & $180-182$ & Gray & $3128(\mathrm{~m})$ & 1226(s) & 3301(s) & $1575(\mathrm{~m})$ & ----- \\
\hline 8 & $\begin{array}{l}{\left[\mathrm{Mn}_{2}(\mathrm{~L})\left(\mathrm{H}_{2} \mathrm{O}\right)_{4}(\gamma-\right.} \\
\left.\mathrm{Pic}_{2} \mathrm{Cl}_{2}\right] \mathrm{Cl}_{2}\end{array}$ & 131 & $240^{*}$ & $\begin{array}{l}\text { Dark } \\
\text { Red }\end{array}$ & $3185(w)$ & 1246(m) & $3371(w)$ & $1628(\mathrm{~m})$ & 1616(s) \\
\hline 9 & $\begin{array}{l}{\left[\mathrm{Fe}_{2}(\mathrm{~L})\left(\mathrm{H}_{2} \mathrm{O}\right)_{4}(\gamma-\right.} \\
\left.\text { pic })_{2} \mathrm{Cl}_{2}\right] \mathrm{Cl}_{2}\end{array}$ & 141 & $198-200$ & Brown & $3175(\mathrm{~m})$ & $1178(\mathrm{~m})$ & $3368(\mathrm{~m})$ & 1634(s) & $1618(\mathrm{~s})$ \\
\hline 10 & $\begin{array}{l}{\left[\mathrm{Co}_{2}(\mathrm{~L})\left(\mathrm{H}_{2} \mathrm{O}\right)_{4}(\gamma-\right.} \\
\left.\text { pic })_{2} \mathrm{Cl}_{2}\right] \mathrm{Cl}_{2}\end{array}$ & 143 & $200^{*}$ & Green & $3190(w)$ & $1273(\mathrm{~s})$ & $3307(\mathrm{~m})$ & $1629(\mathrm{~m})$ & $1608(\mathrm{~m})$ \\
\hline 11 & $\begin{array}{l}{\left[\mathrm{Ni}_{2}(\mathrm{~L})\left(\mathrm{H}_{2} \mathrm{O}\right)_{4}(\gamma-\right.} \\
\left.\mathrm{Pic})_{2} \mathrm{Cl}_{2}\right] \mathrm{Cl}_{2}\end{array}$ & 158 & $230^{*}$ & $\begin{array}{l}\text { Gray } \\
\text { Green }\end{array}$ & $3151(\mathrm{~s})$ & $1252(\mathrm{~s})$ & 3314(s) & $1623(\mathrm{~m})$ & $1618(s)$ \\
\hline 12 & $\begin{array}{l}{\left[\mathrm{Cu}_{2}(\mathrm{~L})\left(\mathrm{H}_{2} \mathrm{O}\right)_{4}(\gamma-\right.} \\
\left.\text { pic })_{2} \mathrm{Cl}_{2}\right] \mathrm{Cl}_{2}\end{array}$ & 135 & $182-184$ & Brown & $3195(\mathrm{~s})$ & $1252(\mathrm{~m})$ & $3342(\mathrm{~m})$ & 1604(m) & $1623(\mathrm{~m})$ \\
\hline 13 & $\begin{array}{l}{\left[\mathrm{Zn}_{2}(\mathrm{~L})\left(\mathrm{H}_{2} \mathrm{O}\right)_{4}(\gamma-\right.} \\
\left.\text { pic })_{2} \mathrm{Cl}_{2}\right] \mathrm{Cl}_{2}\end{array}$ & 147 & $220 *$ & Pink & $3140(\mathrm{~m})$ & $1213(\mathrm{~s})$ & $3269(\mathrm{~s})$ & 1633(s) & 1618(s) \\
\hline 14 & $\begin{array}{l}{\left[\mathrm{Cd}_{2}(\mathrm{~L})\left(\mathrm{H}_{2} \mathrm{O}\right)_{4}(\gamma-\right.} \\
\left.\text { pic })_{2} \mathrm{Cl}_{2}\right] \mathrm{Cl}_{2}\end{array}$ & 167 & $300 *$ & $\begin{array}{l}\text { Light } \\
\text { Gray }\end{array}$ & $3132(\mathrm{~s})$ & $1227(\mathrm{~s})$ & 3303(s) & $1576(\mathrm{~s})$ & $1622(\mathrm{~m})$ \\
\hline
\end{tabular}

*Decomposition , $\gamma$-Pic $=(\mathrm{C}=\mathrm{C}),(\mathrm{C}=\mathrm{N})$ 
Table 4. The \% of C.H.N.Cl analysis for prepared compound, caluculed, (found).

\begin{tabular}{|c|c|c|c|c|}
\hline Number & C\% & H\% & N\% & $\mathrm{Cl} \%$ \\
\hline $\mathrm{L}$ & $\begin{array}{l}\begin{array}{l}64.41 \\
(64.38)\end{array}\end{array}$ & $\begin{array}{l}6.08 \\
(6.04)\end{array}$ & $\begin{array}{l}18.78 \\
(18.71)\end{array}$ & ----- \\
\hline 1 & ----- & ----- & ----- & $\begin{array}{l}22.80 \\
(22.55)\end{array}$ \\
\hline 2 & $\begin{array}{l}30.80 \\
(30.77)\end{array}$ & $\begin{array}{l}4.20 \\
(4.14)\end{array}$ & $\begin{array}{l}8.98 \\
(8.92)\end{array}$ & $\begin{array}{l}22.73 \\
(22.43)\end{array}$ \\
\hline 3 & ---- & ----- & ----- & $\begin{array}{l}22.51 \\
(22.31)\end{array}$ \\
\hline 4 & $\begin{array}{l}30.52 \\
(30.27)\end{array}$ & $\begin{array}{l}4.16 \\
(4.07)\end{array}$ & $\begin{array}{l}8.90 \\
(8.83)\end{array}$ & $\begin{array}{l}22.52 \\
(22.28)\end{array}$ \\
\hline 5 & ----- & ----- & ----- & $\begin{array}{l}22.18 \\
(22.01)\end{array}$ \\
\hline 6 & $\begin{array}{l}29.89 \\
(29.82)\end{array}$ & $\begin{array}{l}4.08 \\
(3.98)\end{array}$ & $\begin{array}{l}8.71 \\
(8.66)\end{array}$ & $\begin{array}{l}22.05 \\
(21.87)\end{array}$ \\
\hline 7 & ----- & ----- & ----- & $\begin{array}{l}19.24 \\
(18.95)\end{array}$ \\
\hline 8 & ----- & ----- & ----- & $\begin{array}{l}17.54 \\
(17.35)\end{array}$ \\
\hline 9 & $\begin{array}{l}41.51 \\
(41.39)\end{array}$ & $\begin{array}{l}4.98 \\
(4.91)\end{array}$ & $\begin{array}{l}10.37 \\
(10.30)\end{array}$ & $\begin{array}{l}17.50 \\
(17.32)\end{array}$ \\
\hline 10 & ----- & ----- & ----- & $\begin{array}{l}17.37 \\
(17.09)\end{array}$ \\
\hline 11 & ----- & ----- & ----- & $\begin{array}{l}17.38 \\
(17.15)\end{array}$ \\
\hline 12 & ----- & ----- & ----- & $\begin{array}{l}17.18 \\
(16.88)\end{array}$ \\
\hline 13 & $\begin{array}{l}40.55 \\
(40.25)\end{array}$ & $\begin{array}{l}4.86 \\
(4.75)\end{array}$ & $\begin{array}{l}10.13 \\
(10.07)\end{array}$ & $\begin{array}{l}17.10 \\
(16.75)\end{array}$ \\
\hline 14 & ----- & ----- & ----- & $\begin{array}{l}15.36 \\
(15.14)\end{array}$ \\
\hline
\end{tabular}

\section{Results and discussion}

$\left.\mathrm{M}_{2}(\mathrm{~L})\left(\mathrm{H}_{2} \mathrm{O}\right)_{4} \mathrm{Cl}_{4}\right]$ and $\left[\mathrm{M}_{2}(\mathrm{~L})(\gamma-\mathrm{Pic})_{2}\left(\mathrm{H}_{2} \mathrm{O}\right)_{4} \mathrm{Cl}_{2}\right] \mathrm{Cl}_{2}$ were characterized using IR, UV - Vis, spectra, magnetic susceptibleted, molar conductivities measurements. The conductivity measurement in $10^{-3} \mathrm{M}$ DMF at $25^{\circ}$ suggesting the non electrolyte for (1-7) complexes and (1:2) electrolytic for (8-14) complexes.

\section{Infrared spectral studies}

The infrared spectrum of (L) ligand showed new bands in the range $\left(3197,3373 \mathrm{~cm}^{-1}\right)$ which are corresponding to $v(\mathrm{NH})$ (Keypour et al., 2002; Chandra and Kumar, 2005) and $v\left(\mathrm{NH}_{2}\right)$ (Dolaz et al., 2001) amine. $\delta(\mathrm{N}-\mathrm{H})$ appears at $\left(1311 \mathrm{~cm}^{-1}\right)$ (Chandra et al., 2004; Siddiqi et al., 2009), and the frequency assignable to $v(\mathrm{C}=\mathrm{O})$ band at $\left(1635 \mathrm{~cm}^{-1}\right)$ (Dey and Ray, 1974; Aggarwal and Narang, 1973).

The position of $v(\mathrm{NH})$ stretching vibration has been shifted to lower frequencies in complexes while ( $\gamma$-Pic) appears at $\left(1608-1623 \mathrm{~cm}^{-1}\right)$ indicating of coordination of $\mathrm{N}$ of $\gamma$-picoline with metal ions (Ibrahim et al., 2014) (Table 3).

\section{Electronic spectra and magnetic measurements}

The magnetic moment values for the $\mathrm{Mn}(\mathrm{II})$ complexes $(1,8)$ have a magnetic moment values $(5.85,5.86$ B.M) (Chandra et al., 2004; Ibrahim et al., 2014) while the electronic band at $(31055,32051,28571,37037) \mathrm{cm}^{-1}$ referring to charge translation range, indicating octahedral geometries structure (Satpute et al., 2015; Kheiri et al., 1977).

The magnetic moment values for the $\mathrm{Fe}$ (II) complexes $(2,9)$ have a values $(4.80,4.91$ B.M) (Shakir et al., 1995; Manzur, 1968) while the electronic band a $(11235,12820) \mathrm{cm}^{-1}$ are attributed ${ }^{5} \mathrm{~T}_{2} \mathrm{~g} \rightarrow{ }^{5} \mathrm{Eg}$ transition indicating octahedral structure (Shakir et al., 1995). 
The magnetic moment for low spin Co(II) complex(3) has (2.48 B.M) value and electronic transition band at (14947) $\mathrm{cm}^{-1}$ which referring to $\left({ }^{2} \mathrm{Eg} \rightarrow{ }^{2} \mathrm{~T}_{2} \mathrm{~g}\right)$. The data suggesting an octahedral geometrical for the complex

(Kataoka and Kon, 1968).

The (high spin) $\mathrm{Co}(\mathrm{II})$ complex, (10) hasamagnetic moment value (4.85) (Nasman, 2001). The UV-VisSpectrum exhibits three bands at $(10928,14925,18315)$ $\mathrm{cm}^{-1}$ which corresponding to $\left(\left({ }^{4} \mathrm{~T}_{1} \mathrm{~g}(\mathrm{~F}) \rightarrow{ }^{4} \mathrm{~T}_{2} \mathrm{~g}\right),\left({ }^{4} \mathrm{~T}_{1} \mathrm{~g}(\mathrm{~F})\right.\right.$ $\left.\rightarrow{ }^{4} \mathrm{~A}_{2} \mathrm{~g}\right),\left({ }^{4} \mathrm{~T}_{1} \mathrm{~g}(\mathrm{~F}) \rightarrow{ }^{4} \mathrm{~T}_{1} \mathrm{~g}(\mathrm{p})\right)$ (Manzur, 1968).

According to mentioned data octahedral geometrical shape has been suggested for complex.

The magnetic moment values for the Ni(II) complexes $(4,11)$ have a values $(2.94,3.06$ B.M.) while three bands at $(9389,18656,24509) \mathrm{cm}^{-1},(9541,14814,24271)$ $\mathrm{cm}^{-1},\left({ }^{3} \mathrm{~A}_{2} \mathrm{~g} \rightarrow \mathrm{v}_{1}{ }^{3} \mathrm{~T}_{2} \mathrm{~g}\right),\left({ }^{3} \mathrm{~A}_{2} \mathrm{~g} \rightarrow \mathrm{v}_{2}{ }^{3} \mathrm{~T}_{1} \mathrm{~g}\right),\left({ }^{3} \mathrm{~A}_{2} \mathrm{~g} \rightarrow\right.$ $\left.\mathrm{v}_{3}{ }^{3} \mathrm{~T}_{1} \mathrm{~g}(\mathrm{P})\right)^{(18)}$, transition indicating octahedral structure (Ibrahim et al., 2014).

The magnetic moment values for the $\mathrm{Cu}$ (II) complexes $(5,12)$ have a magnetic moment values $(2.11,1.94$ B.M) (Saha and Gayen, 1983; Massey and Jahnson, 1975) electronic bands at $(20325,16233,) \mathrm{cm}^{-1}$ which are attributed to and ${ }^{2} \mathrm{Eg} \rightarrow{ }^{2} \mathrm{~T}_{2} \mathrm{~g}$ transition indicating octahedral structure (Raman et al., 2010; Alaghaz and Ammar, 2010).

The $\mu$ eff values of the two $\mathrm{Zn}$ (II) complexes were diamagnetic as expected and the $\mu$ eff values of the two $\mathrm{Cd}(\mathrm{II})$ complexes were diamagnetic as expected (Raman et al., 2010; Kokamota and Hidaka, 1994; Al-Mogren and Alaghaz, 2013).

The conductivity measurements for all the complexes assist the structures which were non-conductive and 1:2 molar ratio (Table 3)

\section{Conclusion}

The present work includes the preparation of new ligand (L) and it's complexes with $\mathrm{Mn}(\mathrm{II}), \mathrm{Fe}(\mathrm{II}), \mathrm{Co}(\mathrm{II}), \mathrm{Ni}(\mathrm{II})$, $\mathrm{Cu}(\mathrm{II}), \mathrm{Zn}(\mathrm{II}), \mathrm{Cd}(\mathrm{II})$ ions, where $\mathrm{L}=\mathrm{N}^{1}, \mathrm{~N}^{4}$ - bis (4amino phenyl) succinamide the characterization of the prepared compounds were carried out on the basis of IR,UV-Visible, spectra, molar conductivity, magnetic susceptibility and micro element analysis measurements. The results suggesting geometrical shapes for all complexes, in which of the ligand coordinates the metal ion via throw $\mathrm{O}(\mathrm{C}=\mathrm{O})$ and $\mathrm{N}\left(\mathrm{NH}_{2}\right)$.

\section{Conflict of interest statement}

Authors declare that they have no conflict of interest.

\section{Acknowledgement}

The authors are thankful to the chemistry Department. College of Education university of Salahaddin for their accomplishing our present work, Dicle university in Turkey for C.H.N and University of Mosul for their helping in IR , magnetic susceptibility measurement.

\section{References}

Aggarwal, R. R., Narang, K. K., 1973. N-acetyl, N'benzoyl hydrazine complexes of cobalt(II), nickel(II) and copper(II)Inorg. Chim. Acta. 7, 651.

Akine, S., Taniguchi, T., Nabeshima, T., 2001. Synthesis and crystal structure of a novel triangular macrocyclic molecule, tris $\left(\mathrm{H}_{2} \mathrm{saloph}\right)$, and its water complex. Tetrahedron Lett. 42, 8861-8864.

Alaghaz, A. M. A., Ammar, R. A., 2010. New dimeric cyclodiphosph(V)azane complexes of $\mathrm{Cr}(\mathrm{III})$, $\mathrm{Co}(\mathrm{II}), \mathrm{Ni}(\mathrm{II}), \mathrm{Cu}(\mathrm{II})$, and $\mathrm{Zn}(\mathrm{II})$ : Preparation, characterization and biological activity studies. Eur. J. Med. Chem. 45, 1314-1322.

Al-Mogren, M. M., Alaghaz, A-N. M., 2013. Synthesis, spectral and quantum chemical calculations of mononuclear nickel(II), copper(II) and cadmium(II) complexes of new Schiff-base ligand. Int. J. Electrochem. Sci. 8, 8669-8685.

Beswick, C.L., Shalders, R.D., Swaddle, T.W., 1996. Volume profile for substitution in labile chromium (III) complexes: Reactions of aqueous [Cr(Hedta) $\left.\mathrm{OH}_{2}\right]$ and [Cr(edta)]- with thiocyanate ion. Inorg. Chem. 35, 991-994.

Chandra, S., Gupta, L.K., Jain, D., 2004. Spectroscopic studies on $\mathrm{Mn}(\mathrm{II}), \mathrm{Co}(\mathrm{II}), \mathrm{Ni}(\mathrm{II})$ and $\mathrm{Cu}(\mathrm{II})$ complexes with $\mathrm{N}$-donor tetradentate $\left(\mathrm{N}_{4}\right)$ macrocyclic ligand derived from ethylcinnamate moiety. Spectrochim. Acta A. 60, 2411.

Chandra, S., Kumar, R., 2005. Synthesis, electrochemistry and spectral studies on Cobalt(II) and Manganese (II) complexes with 12-, 14-, 15-, and 18-membered $\mathrm{N}_{4}, \mathrm{~N}_{2} \mathrm{O}_{2}, \mathrm{~N}_{2} \mathrm{O}_{2} \mathrm{~S}, \mathrm{~N}_{6}$ donor macrocyclic ligands Synth. React. Inorg. Met. Org. Nano-Metal Chem. 35, 161-170.

Christensen, J.J., Hill, J.O., Izatt, R.M., 1971. Ion binding by synthetic macrocyclic compounds. 
Science. 174(4008), 459.

Delgado, R., 1995. Co-ordination chemistry with macrocyclic compounds. Rev. Port. Quim. 2, 18-29.

Dey, K., Ray, K., 1974. Chromium(III) complexes of Schiff bases. Inorg. Chim. Acta.10, 139.

Dolaz, M., Tumer, M., Golcu, A., Serin, S., 2001. Synthesis and spectrophotometric investigation of a new vic-dioxime ligand and its transition metal complexes. Turk. J. Chem. 25, 491-500.

Goedken, V. L., 1979. In: Coordination Chemistry of Macrocyclic Compound (Ed.: Melson, G). Plenum Press, New York. pp.603-654.

Ibrahim, O.B., Mohamed, M.A., Rafat, M.S., 2014. Can. Chem. Transact. 2(2), 108-121.

Kataoka, N., Kon, H., 1968. A hexacoordinated lowspin cobalt(II) complex. J. Am. Chem. Soc. 90(11), 2978-2979.

Keypour, H., Salehzadeh, S., Parish, R. V., 2002. Synthesis of two potentially heptadentate $\left(\mathrm{N}_{4} \mathrm{O}_{3}\right)$ Schiff-base ligands derived from condensation of tris(3-aminopropyl)-amine and salicylaldehyde or 4hydroxysalicylaldehyde. Nickel(II) and Copper(II) complexes of the former ligand. Molecules. 7, 140144.

Kheiri, F. M. N., Tsipis, C. A., Manoussakis, G. E., 1977. Syntheses and spectroscopic study of some new mixed-ligand $\mathrm{Bi}$ (III) 1,1-dithiolate complexes. Inorg. Chem. Acta , 25 , 223-227.

Konno, T., Okamoto, K., Hidaka, J., 1994. Synthesis and properties of T-cage-type S-bridged rhodium(III) zinc(II) octanuclear complexes with 2aminoethanethiolate of L-cysteinate. Inorg. Chem. 33, 538-544.

Lindoy, L. F. (Ed.), 1989. Chemistry of Macrocyclic Ligand Complexes. Cambridge University Press, Cambridge, UK.

Manzur, J., 1986. New complexes of iron(II), cobalt(II), nickel(II) and copper(II) with 2,2'-dipyridyl - methane. Trans. Met. Chem. 11, 220-223..

Massey, A.C., Jahnson, B.F.G., 1975. The Chemistry of Copper, Silver and Gold. Pergamon Press, Oxford.41p.

Melson, G., 1979. Coordination Chemistry of Macrocyclic Compounds, Plenum Press, New York. pp.1-16.

Nasman, O.S.M., 2001. Binuclear $\mathrm{N}_{8}$ 28-membered macrocyclic transition metal complexes: Synthesis and characterization. Synth. React. Inorg.Met.-Org. Chem. 31(8), 1433-1442.

Raman, N., Selvan, A., Manisankar, P., 2010. Spectral, magnetic, biocidal screening, DNA binding and photocleavage studies of mononuclear $\mathrm{Cu}(\mathrm{II})$ and $\mathrm{Zn}$ (II) metal complexes of tricoordinate heterocyclic Schiff base ligands of pyrazolone and semicarbazide/thiosemicarbazide based derivatives. Spectrochim. Acta A, 76, 161-173.

Saha, N., Gayen, N.C., 1983. Mixed-ligand complexes of cobalt(II), nickel(II) and copper(II) with 1guanyl-5-methyl pyrazole-3-carboxylic acid. J. Ind. Chem. Soc. 60, 317.

Satpute, R.H., Dhokte, A. O., Sakhare, M.A., Arbad, B.R., 2015. Spectroscopic studies on Cr(III), Mn(II) and $\mathrm{Zr}(\mathrm{IV})$ complexes with $\mathrm{N}$-donortetradentate (N4) macrocyclic ligand. Der Chem. Sinica. 6(5), 36-41.

Shakir, M., Mohamed, A.K., Varkey, S.P., Nasman, O.S.M., Siddiqi, Z.A., 1995. Preparation and structural characterization of 14-16-membered pendent arm macrocyclic complexes of transition metal ions. Polyhedron. 14(10), 1277-1282.

Siddiqi, Z.A., Kumar, S., Khalid, M., Shahid, M., 2009. Synthesis and spectral investigations of mononuclear [N6], [N8] and dinuclear [N10] complexes of polyamide macrocycles: 57Fe Mossbauer parameters of $\mathrm{Fe}(\mathrm{III})$ complexes. Spectrochim. Acta A. 72, 616-620.

\section{How to cite this article:}

Ali Mohamad, H., Salahaddin Shreef, H., 2017. Synthesis and characterization of some transition and non-transition metal complexes with new cyclic and open series containing (N, O) as donor atoms. Int. J. Curr. Res. Biosci. Plant Biol. 4(8), 15-21. doi: https://doi.org/10.20546/ijcrbp.2017.408.003 